\title{
Challenges and unanswered questions for the next decade of immune-oncology research in NSCLC
}

\author{
Niki Karachaliou $^{1,2}$, Manuel Fernandez-Bruno ${ }^{1}$, Jillian Wilhelmina Paulina Bracht ${ }^{2}$, Rafael Rosell ${ }^{2,3,4,5}$ \\ ${ }^{1}$ Institute of Oncology Rosell (IOR), University Hospital Sagrat Cor, QuironSalud Group, Barcelona, Spain; ${ }^{2}$ Pangaea Oncology, Laboratory of \\ Molecular Biology, Quiron-Dexeus University Institute, Barcelona, Spain; ${ }^{3}$ Institute for Health Science Research Germans Trias i Pujol (IGTP), \\ Badalona, Spain; ${ }^{4}$ Institute of Oncology Rosell (IOR), Quiron-Dexeus University Institute, Barcelona, Spain; ${ }^{5}$ Catalan Institute of Oncolgy, Hospital \\ Germans Trias i Pujol, Badalona, Spain \\ Contributions: (I) Conception and design: N Karachaliou, R Rosell ; (II) Administrative support: All authors; (III) Provision of study materials or \\ patients: N Karachaliou, M Fernandez-Bruno; (IV) Collection and assembly of data: N Karachaliou, M Fernandez-Bruno; (V) Data analysis and \\ interpretation: All authors; (VI) Manuscript writing: All authors; (VII) Final approval of manuscript: All authors. \\ Correspondence to: Niki Karachaliou. Institute of Oncology Rosell (IOR), University Hospital Sagrat Cor, QuironSalud Group, Viladomat 288, \\ Barcelona 08017, Spain. Email: nkarachaliou@oncorosell.com.
}

\begin{abstract}
Over the last 20 years there have been great advances in the treatment of lung cancer. Immune checkpoint blockade together with targeted therapies have provided oncologists with the means to improve survival of non-small cell lung cancer (NSCLC) and patients with a better quality of life and therapies with manageable toxicity. Maybe in a short period of time the possibility of a cure in metastatic NSCLC will be raised. Therefore, continued research into new drugs, biomarkers and especially combination therapies is necessary in order to expand the clinical benefit of the current treatments to a broader population of NSCLC patients. The purpose of our review is to highlight our thoughts about potential mechanisms of resistance to immunotherapy that, if better explored, can provide us with both biomarkers to predict response to these therapies and partners to combine with and prolong the benefit of immune checkpoint blockade. We are presenting our own experience of immunotherapy with a case report from our institution.
\end{abstract}

Keywords: Lung cancer; immunotherapy; biomarkers; combinations

Submitted Apr 04, 2018. Accepted for publication Jun 25, 2018.

doi: $10.21037 /$ tlcr.2018.06.08

View this article at: http://dx.doi.org/10.21037/tlcr.2018.06.08

\section{Introduction}

The concept of cancer immunotherapy is not new, it goes back to 1891 but it was not successful until recently, when the existence and role of immune checkpoints for cancer were understood (1). The role of immune checkpoints like programmed cell death protein-1 (PD-1) or cytotoxic T-lymphocyte associated protein 4 (CTLA-4) is to reduce autoimmune responses against self-tissues. The immune checkpoint PD-1, expressed on $\mathrm{CD}^{+}$and $\mathrm{CD}^{+}{ }^{+} \mathrm{T}$ cells and B cells, was discovered in 1992 by Japanese investigators (2-4). Almost 10 years later, the same group of investigators discovered the ligands PD-L1 (5) and PD-L2 (6). Both are type I transmembrane proteins. PD-L1 is expressed in lymphoid and non-lymphoid tissues, including various tumor cells and virus-infected cells, while PD-L2 is expressed only on antigen-presenting cells, like dendritic cells and macrophages $(7,8)$. Nivolumab (also known as ONO4538, MDX-1106 or BMS-936558) is the first fully humanized anti-PD-1 monoclonal antibody, developed in 2005 , using genetically modified mice carrying loci encoding human immunoglobulins (9).

From 2005 until now, several anti-PD-1 or anti-PD-L1 monoclonal antibodies have demonstrated activity in nonsmall cell lung cancer (NSCLC) patients alone, or in combination with other anti-tumor therapies in advanced and more recently in early stage disease (10). The antiPD-1 antibody nivolumab (11) and the anti-PD-L1 
antibody atezolizumab $(12,13)$ are approved for the secondline therapy of advanced stage NSCLC, with no biomarker required for the selection of patients. The anti PD-1 antibody pembrolizumab is approved in the same setting, but only when there is a PD-L1 tumor proportion score of at least $1 \%$. Nivolumab failed in the first-line setting in the CheckMate-026 trial (14) while pembrolizumab secured first-line approval on the basis of the Keynote-021 data (15) for PD-L1 positive patients (tumor proportion score of at least $50 \%)$.

All mentioned compounds have also been tested in other tumors (16) and have obtained approval in different settings, as shown in Figure 1. The last 2 years combination studies with chemotherapy or small molecules have come across the board, with both pembrolizumab and nivolumab being the most widely combined anti-PD-1 agents (17). Pembrolizumab in combination with carboplatin and pemetrexed is approved for the first-line therapy of unselected advanced stage lung adenocarcinoma patients $(18,19)$ (Figure 1). Anti-PD-L1 antibodies like durvalumab and avelumab (20) have demonstrated efficacy in lung and other types of tumors and new compounds like BGB-A317, LY3300054, CX-072, PDR001 and FAZ053 have made their entrance in immune-oncology.

Despite the significant survival benefit of immune checkpoint inhibitors for some patients with advanced NSCLC, overall, the objective response rate is not more than $20-30 \%$ with a large proportion of patients not responding at all to these therapies (16). Still, we are not in a position to select with confidence the patients to treat with immunotherapy. PD-L1 status guides the first-line therapy but this is not the case for the second-line setting (21). A new pattern of progression, named "hyper-progression" has been described for a small but still significant proportion of patients who progress or die within the first 3 months of therapy with immune checkpoint inhibitors (22). The survival benefit of immune checkpoint inhibitors, at least as monotherapy, has not been confirmed in oncogene-addicted tumors, like, for instance, lung adenocarcinomas carrying epidermal growth factor receptor (EGFR) mutations (23).

In this review we will go through signaling pathways that can provide us with biomarkers for response to immunotherapy and can also be relevant for resistance to immune checkpoint blockade therapy. Potential solutions will be commented. The revision of second and third generation drugs for immuno-oncology is out of the scope of our review. Some thoughts about immunotherapy in early stage NSCLC will be provided.

\section{Biomarkers for immunotherapy: the present}

PD-L1 expression is the only accepted biomarker to select patients for immunotherapy but still its performance in discriminating responders from non-responders is debatable. Three immunohistochemistry staining assays are most commonly used (the Ventana SP263, Dako 22C3 and Dako 28-8) (1,24). Caution should be taken with the use of PD-L1 protein expression as an exclusive predictive biomarker, considering the variability of its expression at different sites of disease or at different time points during the treatment course $(1,10)$.

Tumor mutation burden (TMB) is another biomarker of response to immunotherapy across multiple tumor types (25). For instance, although overall the CheckMate-026 was a negative trial, in an exploratory biomarker analysis of TMB by whole-exome sequencing, patients with 243 or more mutations (high TMB) had a higher response rate and a longer progression-free survival with nivolumab in comparison with first-line chemotherapy (14). High TMB can similarly predict efficacy to nivolumab plus ipilimumab in small-cell lung cancer patients, upon progression to first-line therapy (26). Until recently, TMB was assessed only by whole-exome sequencing $(14,27)$, which makes its clinical application complex. Today, targeted next generation sequencing can also be applied to TMB evaluation as a predictor of benefit for immune checkpoint blockade (28). In the recent study of Rizvi et al., targeted next generation sequencing was performed using the Memorial Sloan Kettering Cancer Center-Integrated Mutation Profiling of Actionable Cancer Targets (MSKCC-IMPACT) platform (28). TMB can also be performed using other commercial platforms in both tissue and blood (29). In a retrospective analysis of the CheckMate-012, which evaluated the safety and efficacy of nivolumab plus ipilimumab in the first-line setting of NSCLC patients, TMB as assessed by three methods, whole-exome sequencing, the MSKCC-IMPACT platform and the FoundationOne platform, had similar predictive fidelity for efficacy (30). The cutoff for defining low and high TMB with the FoundationOne panel has been explored in the CheckMate-568 study. A TMB of 10 mutations per megabase was able to discriminate responders from non-responders to the combination of nivolumab plus ipilimumab as presented by Dr. Ramalingam, in the last American Association of Cancer Research meeting (AACR, 2018). For patients with high TMB ( $>10$ mutations per megabase) the combination of nivolumab plus ipilimumab 


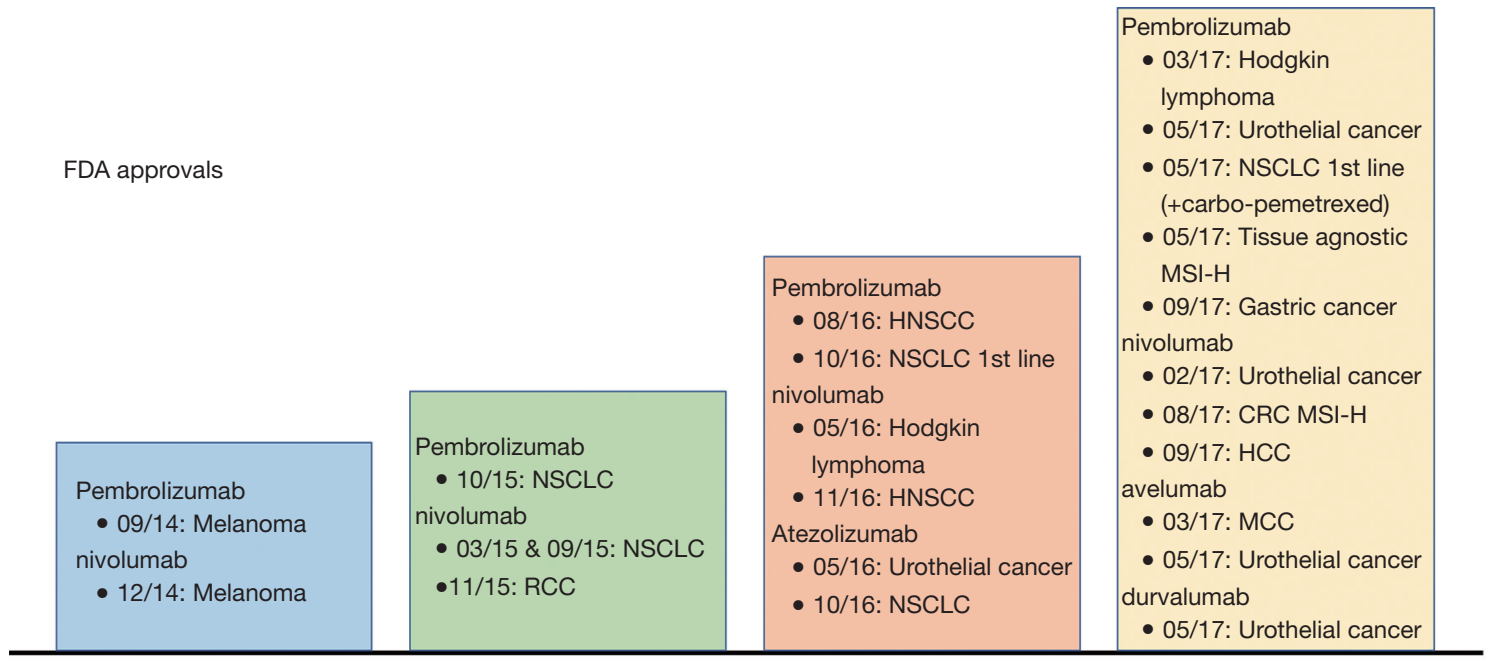

Figure 1 FDA approvals for anti-PD-1 and anti-PD-L1 inhibitors in cancer. FDA, Food and Drug Administration; NSCLC, non-small cell lung cancer; HNSCC, head and neck squamous cell carcinoma; MSI-H, microsatellite instability-high; RCC, renal cell carcinoma; HCC, hepatocellular carcinoma; MCC, Merkel cell carcinoma.

significantly improved the progression-free survival of treatment-naïve metastatic NSCLC patients in comparison with platinum-based chemotherapy (31). With the GUARDANT360 liquid biopsy test, the detection of six or more variants or more than three variants of unknown significance was found to be predictive of response to immunotherapy (32). In addition, with the same test it has been demonstrated that cell-free DNA velocity is an early surrogate of immunotherapy response $(33,34)$. With the FoundationAct 396 gene-based liquid biopsy test, a blood $\mathrm{TMB}$ of more or equal than 16 was found to be predictive of response to atezolizumab (35). Next generation sequencing platforms are also determining microsatellite instability (MSI) status (36). Pembrolizumab is approved for treatment-refractory MSI-high or mismatch repair deficient metastatic solid tumors $(37,38)$ (Figure 1).

PD-L1 expression, TMB and MSI are means to select immunotherapy for cancer patients including NSCLC. In most of the studies, no association has been found among them, resulting in a difficult decision-making process, especially in the second-line therapy of NSCLC. Another challenging question in immunology is how the immune system affects cancer development and progression. The immune system can recognize and kill tumor cells but it can also facilitate tumor progression by establishing a tumor microenvironment that accommodates tumor growth (cancer-immune editing) (39).

\section{Novel biomarkers for immunotherapy and potential combinations: the future}

PD-L1 expression is induced by oncogenic signals or by inflammatory cytokines such as interferon- $\gamma$. We reported interferon- $\gamma$ as a biomarker to predict response to immune checkpoint blockade in melanoma and NSCLC patients receiving nivolumab and pembrolizumab, respectively (40). Indeed, now interferon- $\gamma$ signatures are prospectively validated as a biomarker in ongoing immunotherapy clinical trials (40). In the absence of interferon- $\gamma$ in the tumor microenvironment, CKLF-like MARVEL transmembrane domain containing protein 6 and 4 (CMTM6 and CMTM4) are the only regulators of PD-L1 expression (41). CMTM6 is a transmembrane protein that associates with PD-L1 in the cell surface and protects it from lysosomal degradation (42) (Figure 2). For this function CMTM6 cooperates with its closest family member, CMTM4, but not with other CMTM family members (42). A CMTM6/4 inhibitor is in clinical development (Table 1).

In surgical resected NSCLC specimens, PD-1 (or B7$\mathrm{H} 1)$ is co-expressed with $\mathrm{B} 7-\mathrm{H} 3$ and both molecules repress the antitumor immune response by inhibiting $\mathrm{T}$ cell infiltration and IFN- $\gamma$ secretion (57) (Figure 2). The negative prognostic significance of $\mathrm{B} 7-\mathrm{H} 3$ expression is more evident in smokers and EGFR wild type lung adenocarcinoma patients (58). Combining blockades of B7-H3 and PD-1 enhanced therapeutic tumor control 


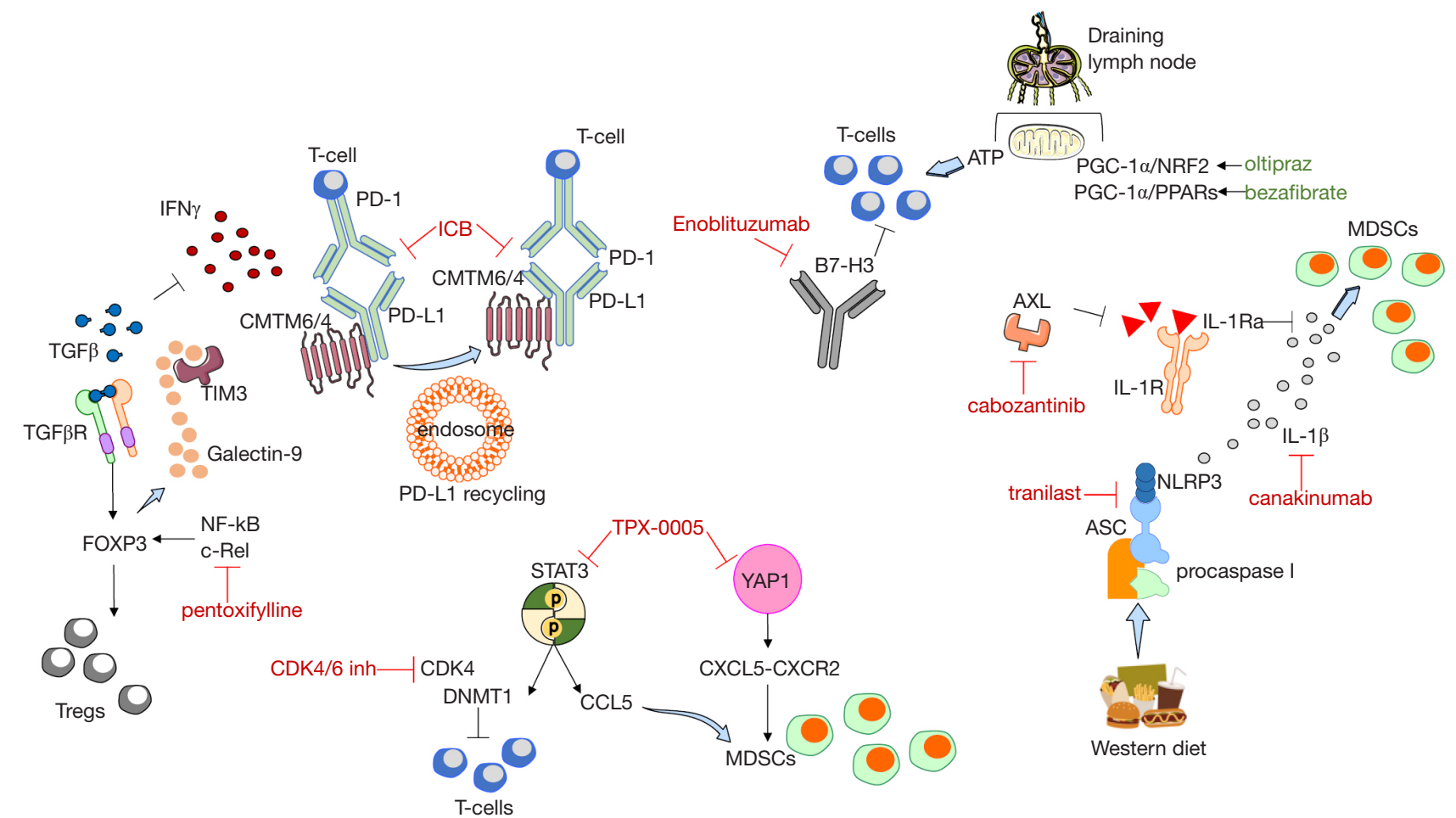

Figure 2 Potential targets to enhance the efficacy of immune checkpoint blockade. Signaling pathways that are related with resistance to anti-PD-1 and anti-PD-L1 therapies and drugs that can be used to abrogate them. ICB, immune checkpoint blockade.

compared to PD-1 inhibition alone, in vivo $(59,60)$. A B7$\mathrm{H} 3$ inhibitor, enoblituzumab has shown promising data in a phase I clinical trial and now is being evaluated in combination with anti-PD-1 therapies (ClinicalTrials.gov identifier NCT02475213) $(43,44)$ (Table 1).

Whether or not there is a positive correlation between EGFR mutations or anaplastic lymphoma tyrosine kinase (ALK) rearrangements and PD-L1 expression is not clear. Several studies have shown that the expression of PD-L1 is higher in EGFR or ALK altered NSCLC in comparison with wild type tumors (61-66) but it is not related with better response to immune checkpoint blockade. Still there are few studies showing decreased expression of PDL1 in EGFR or ALK driven tumors $(67,68)$. We found that patients with high PD-L1 expression have better outcome to EGFR tyrosine kinase inhibitors (TKI) (69). Co-expression of transcriptional regulators, like signal transducer and activator of transcription 3 (STAT3) and yes-associated protein 1 (YAP1) (70) and receptor and nonreceptor tyrosine kinases (RTKs and non-RTKs), mainly AXL and CUB domain-containing protein 1 (CDCP1) (45) are related to worse outcome to EGFR TKIs. This can also partially explain the lack of response of EGFR mutant NSCLC to immune checkpoint inhibitors. Both STAT3 and Src-YAP1 have an immunosuppressive role by either interfering with interferon responses or by inducing the expression of chemokines that attract myeloid-derived suppressor cells (MDSCs) (71). YAP1 plays a key role in the efficient recruitment of MDSCs through activation of the CXCL5-CXCR2 axis (72). In our study, the multi-kinase inhibitor TPX-0005 was able to reverse the EGFR TKIinduced activation of STAT3, Src-YAP1 and RTKs (45) The combination of TPX-0005 with immune checkpoint blockade can be of interest in EGFR mutant NSCLC or other types of tumors (Table 1). After many studies having shown the negative impact of immune checkpoint inhibitors as second line therapy in EGFR or ALK positive NSCLC (73), for the first time, an immunotherapy combinatorial approach, specifically atezolizumab, bevacizumab carboplatin-paclitaxel, demonstrated clinically meaningful progression-free survival benefit in the first-line setting of patients with EGFR or ALK rearrangements (74). Still, whether this complex combinatorial approach will have a clinical impact in the first-line therapy of EGFR or 
Table 1 Combinations of immune checkpoint blockade (ICB) and targeted therapies that merit to be investigated

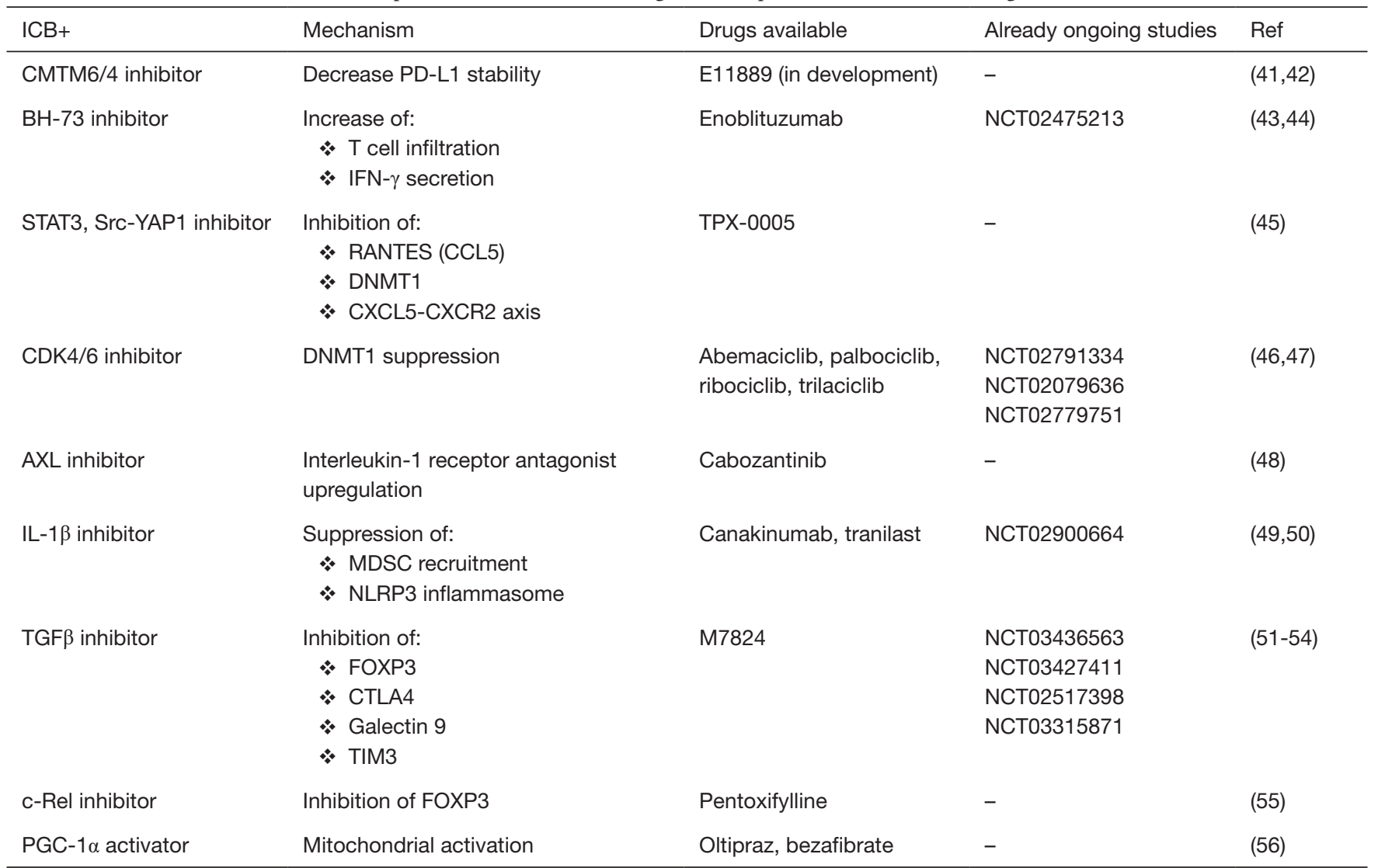

ALK positive NSCLC patients is doubtful.

CC chemokine ligand 5 (CCL5), also known as RANTES (Regulated upon Activation, Normal T-cell Expressed, and Secreted), is a transcriptional target of STAT3 and is related to immune suppression and, ultimately, resistance to immunotherapy $(75,76)$. Among other chemokines, RANTES is important for trafficking MDSCs and regulatory $T$ cells (Tregs) to the tumor (75). Other studies have related RANTES with the recruitment of $\mathrm{CD}^{+}$cells and therefore response to immune checkpoint blockade $(77,78)$. This is probably due to the fact that RANTES attracts a wide range of immune cells and its effect may be differentiated according to the tumor type or even according to the experimental procedures (e.g., in vitro or in vivo studies). STAT3 activates DNA methyltransferase 1 (DNMT1) that impairs T-helper 1 cytokine production and responsiveness to checkpoint blockade $(79,80)$ (Figure 2). Cyclin-dependent kinase 4 (CDK4) interacts with DNMT1 and the combination of immune checkpoint inhibitors with CDK4/6 inhibitors was found to be synergistic in vitro and in vivo $(46,47)$ (Figure 2). Clinical trials are now ongoing with this combination (ClinicalTrials. gov identifiers NCT02791334, NCT02079636, and NCT02779751) and could be of important benefit for tumors with poor outcomes to current immunotherapies like EGFR mutant NSCLC.

On the other hand, AXL matters for tumors that do not derive benefit from immune checkpoint blockade, like prostate cancer (81). AXL and other mesenchymal transition genes are related with innate resistance to antiPD-1 therapy (82). In mice models of castration-resistant prostate cancer, immunotherapy alone induced the AXL expression and only when a combination of immunotherapy with cabozantinib (MET, VEGFR2, AXL and RET inhibitor) was administered, RTKs, including AXL, were downregulated, while interleukin-1 receptor antagonist (IL-1Ra) was upregulated (48). IL-1Ra suppresses MDSC recruitment (83) (Figure 2). IL-1Ra is a regulator of $\mathrm{IL}-1 \beta$ signaling. Canakinumab, an anti-inflammatory therapy for atherosclerotic disease, is a human anti-IL-1 $\beta$ monoclonal 
antibody (84). IL-1 $\beta$ inhibition with canakinumab therapy is also related to reduction in lung cancer mortality and reduction in lung cancer cases in patients with previous heart attack and inflammatory atherosclerosis (49). A study with the combination of the anti PD-1 antibody spartalizumab (PDR001) with canakinumab is ongoing (ClinicalTrials.gov identifiers NCT02900664).

Host-related influences may be responsible for the heterogeneous responses and failures during immune checkpoint blockade therapies. A strong interrelationship has been described between the host microbiota and immunotherapy (85). Inflammasome is an innate immune pathway, responsible for the production of the inflammatory cytokine IL-1 $\beta$ (Figure 2). In response to danger signals like bacterial or viral infections or inflammatory diseases, NOD-like receptors (NLR) interact with adaptor molecule apoptosis-associated speck-like protein (ASC) and procaspase I to form the inflammasome (86). NLRP3 inflammasome is the best characterized inflammasome. There is evidence that NLRP3 activation inhibits antitumor immune response by augmenting the function of immunosuppressive cells like MDSCs (87) (Figure 2). Western diet triggers NLRP3 innate immune reprogramming, which may also explain the clinical benefit of canakinumab in humans with cardiovascular risk $(49,84,88)$. Tranilast, an old anti-allergic clinical drug, is a direct NLRP3 inhibitor (50) (Table 1).

Transforming growth factor- $\beta$ (TGF $\beta$ ) signaling is related to immunosuppression and restriction of T-cell infiltration. TGF $\beta$ reduces the expression of interferon- $\gamma$, attenuates the activity of $\mathrm{CD}^{+} \mathrm{T}$ cells and, more importantly, induces the expression of the transcription factor Forkhead box P3 (FOXP3) (Figure 2). FOXP3, among other functions, is responsible for the differentiation of Tregs and the maintenance of their signaling. Furthermore, the TGF $\beta$ FOXP3 axis stimulates the expression of CTLA-4 and galectin 9, the ligand of the immune-inhibitory receptor T-cell immunoglobulin domain and mucin domain-3 (TIM-3) (51) (Figure 2). The combination of anti-PD-1/ PD-L1 antibodies with drugs that block TGF $\beta$ was highly synergistic in microsatellite-stable colon (52) and urothelial (53) cancer and enabled immune infiltration. M7824, a bifunctional fusion protein targeting PD-L1 and TGF- $\beta$, is now in clinical trials (ClinicalTrials.gov identifiers NCT03436563, NCT03427411, NCT02517398 and NCT03315871) (54). FOXP3 and Tregs development is also dependent on c-Rel, a subunit of the canonical nuclear factor $\kappa$-light-chain-enhancer of activated $\mathrm{B}$ cells $(\mathrm{NF}-\kappa \mathrm{B})$ pathway (55) (Figure 2). Pentoxifylline is an FDA-approved drug, already in the market, used in patients to increase blood flow in the hands and feet of people with poor circulation. It also causes c-Rel degradation and impairs Treg identity (55). Of course, caution should be taken since the destruction of Tregs can generate autoimmune reactions.

\section{Immunotherapy for early stage NSCLC: adjuvant or neoadjuvant?}

Immunotherapy in lung cancer has demonstrated significant activity in early stage disease (89). When Rafael Rosell was asked about the PACIFIC study, he commented that the results undeniably point to implementing immunotherapy with chemoradiotherapy in patients with stage III NSCLC. In addition, he said that the study prompts us to gain further insights on the immune effects of irradiation (90). Surprisingly, two administrations of nivolumab before surgical resection of stage I-IIIA NSCLC was associated with a major pathological response in $45 \%$ of resected tumors (91). Whether the effect of immunotherapy can be stronger in the neoadjuvant setting compared to after dissection of lymph nodes, which can reduce the antitumor activity of the immune system, merits further investigation in lung cancer $(56,92)$. In breast cancer mice models, neoadjuvant immunotherapy had a significant better therapeutic efficacy in comparison with adjuvant therapy (93).

The rapid proliferation and differentiation of $\mathrm{T}$ cells is necessary for an effective PD-1 blockade therapy. Immune metabolism requires mitochondria in draining lymph nodes to supply tumor-reactive cytotoxic $\mathrm{T}$ lymphocytes with ATP, but the preexisting cytotoxic T lymphocytes are not enough for durable tumor-growth inhibition. Indeed, draining lymph node ablation cancels the efficacy of the PD-1 blockade therapy (56). PPAR-gamma coactivator $1 \alpha$ (PGC-1 $\alpha$ ) enhances mitochondrial activity through partner transcription factors: nuclear respiratory factors (NRFs) and peroxisome proliferator-activated receptors (PPARs) (56). Oltipraz and bezafibrate are small-molecule activators of PGC- $1 \alpha / \mathrm{NRF} 2$ and PGC- $1 \alpha /$ PPARs, respectively, and have demonstrated synergistic tumor suppression activity with anti-PD-L1 monoclonal antibodies in animal models (56). 

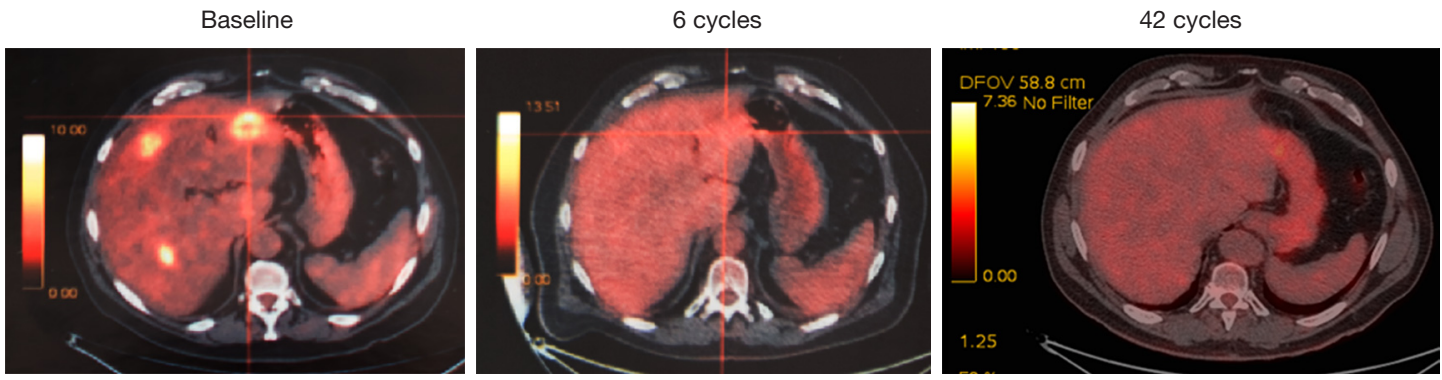

\begin{tabular}{|l|c|c|c|c|}
\hline PET CT $(\mathrm{SUV})$ & LUNG & LIVER & T12 & $4^{\text {th }}$ right rib \\
\hline 21/4/16 basal & 6.7 & 14.6 & 8.1 & 10.9 \\
\hline 11/8/16 6 cycles & 4 & -- & 4.6 & 2.9 \\
\hline $3 / 11 / 1612$ cycles & 2 & -- & 2.1 & 1.9 \\
\hline $3 / 2 / 1718$ cycles & 1.9 & -- & 1.8 & 1.5 \\
\hline $7 / 4 / 1722$ cycles & 1.7 & -- & -- & -- \\
\hline $20 / 10 / 1730$ cycles & 2 & -- & -- & -- \\
\hline $20 / 1 / 1842$ cycles & 2 & -- & -- & -- \\
\hline
\end{tabular}

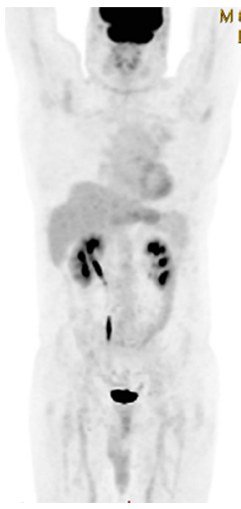

Figure 3 Long-term survival of a patient with metastatic squamous cell NSCLC treated with second-line nivolumab. Upper part: metastatic localizations in the liver in the PET-CT-scan of April 2016 (on the left), before treatment with nivolumab, August 2016, after 6 cycles of treatment with nivolumab (on the middle), and January 2018, after 42 cycles of treatment with nivolumab (on the right). Lower part: the table on the left shows the metabolic activity of the disease during the treatment. The whole-body PET study (on the right) is of January 2018, after 42 cycles of treatment with nivolumab. SUV, standardized uptake value; T12, twelfth thoracic vertebra.

\section{Our experience and future perspectives}

Immunotherapy constitutes a milestone in the advancement of lung cancer treatment. Until now, we could never consider that a $16 \% 5$-year overall survival rate could occur in heavily pretreated metastatic NSCLC patients (94). We report the case of a 62-year-old male patient who arrived to our institution in April 2016, with a metastatic squamous cell carcinoma of the lung and progression to first-line therapy. The patient was initially diagnosed in October, 2015, with a squamous cell carcinoma of the lung and brain metastases. He was treated in his local hospital with four cycles of cisplatin with paclitaxel, consolidation radiotherapy of the lung and the mediastinum and radiosurgery of the brain metastases. Only three months after the end of the treatment, he progressed with new liver and bone metastases. At that time the patient came to us with a performance status of 2 for a second opinion. We initiated second-line therapy with nivolumab $3 \mathrm{mg} / \mathrm{kg}$ every 2 weeks. There was no tissue available for molecular analysis and we planned to perform a biopsy of the liver metastases if the performance status of the patient improved with the treatment. An impressive clinical, radiographic and metabolic response, with complete disappearance of the liver metastases was observed after only 6 administrations of nivolumab. The patient is still radiographically free of disease (after 42 cycles), 2 years and 6 months after being diagnosed with metastatic NSCLC. We never had the opportunity to perform a rebiopsy. The patient continues therapy with nivolumab without any significant toxicity (Figure 3). Immune related toxicities are out of the scope of our review, but there are several reports and guidelines that are useful for oncologists and hospitalists who are treating patients receiving immune checkpoint inhibitors $(95,96)$.

In the first-line setting of NSCLC patients without driver genetic alterations, treatment decisions have become more complex than in the past. The results of the CheckMate-227 showed that nivolumab plus ipilimumab is an effective treatment option for patients with high TMB. Still, there are several challenges, such as the reliability of the metrics to identify patients with high TMB or the tissue requirements for such analysis that usually surpasses the 
quantity of material that we obtain by performing small biopsies. Furthermore, the turnaround time of a targeted next generation sequencing cannot be less than 10 days, the cost of the test is significantly high and it is not easily available in all hospitals. In the CheckMate-227, patients were not randomized by TMB and the overall survival results are relatively immature with a non-significant trend toward improved overall survival for patients with high TMB receiving the immunotherapy combination. On the other hand, we have the results of the Keynote-189 in which the benefit in progression-free and overall survival of pembrolizumab combined with platinum-based chemotherapy extended across all PD-L1 levels, even patients with low PD-L1 expression. Last but not least we should not forget that pembrolizumab alone has comparable efficacy to pembrolizumab combined with platinum-based chemotherapy, has lower risks for side-effects and preserves the option of platinum-based chemotherapy upon disease progression, for patients with high PD-L1 (97).

Finally, many ongoing trials combine anti-PD-1/PD-L1 monoclonal antibodies with chemotherapy, radiotherapy, targeted therapies or other immune checkpoint inhibitors. Looking at the results of the most relevant clinical trials comparing immunotherapy with chemotherapy $(11,15)$, we observe that survival curves separate at later times (98). However, this is not the case for studies in which immunotherapy is combined with other therapies like chemotherapy or radiotherapy $(89,99)$. When $20 \%$ of early events were excluded, more significant $\mathrm{P}$ values and more pronounced estimates of the treatment effect were theoretically generated (98). This could be due to the fact that the generation of an adaptive immune response in patients with rapidly progressive disease (100) may be difficult without the combination of immune checkpoint blockade with other therapies, at least for a period of time. Whether the best partner for immunotherapy is chemotherapy, radiotherapy, other immune checkpoint inhibitors, or drugs that target compensatory signaling pathways is now under active investigation. In Table 1 we provide some rational combinations and biomarkers that may become clinically applicable in the near future, while Figure 2 graphically explains these pathways.

\section{Acknowledgements}

Funding: Work in Dr. Rosell's laboratory is partially supported by a grant from La Caixa Foundation, an Instituto de Salud Carlos III grant (RESPONSE,
PIE16/00011) and a Marie Skłodowska-Curie Innovative Training Networks European Grant (ELBA No. 765492). The work of Jillian Wilhelmina Paulina Bracht in Pangaea Oncology is supported by a Marie Skłodowska-Curie Innovative Training Networks European Grant (ELBA No. 765492). The funders did not have any role in the writing of the review.

\section{Footnote}

Conflicts of Interest: The authors have no conflicts of interest to declare.

\section{References}

1. Karachaliou N, Rosell R. Science and biology drives the immune system to cure lung cancer patients: a revolution but not without challenges. Ther Adv Med Oncol 2018;10:1758835918763723.

2. Ishida Y, Agata Y, Shibahara K, et al. Induced expression of PD-1, a novel member of the immunoglobulin gene superfamily, upon programmed cell death. EMBO J 1992;11:3887-95.

3. Agata Y, Kawasaki A, Nishimura H, et al. Expression of the PD-1 antigen on the surface of stimulated mouse $\mathrm{T}$ and B lymphocytes. Int Immunol 1996;8:765-72.

4. Iwai Y, Okazaki T, Nishimura H, et al. Microanatomical localization of PD-1 in human tonsils. Immunol Lett 2002;83:215-20.

5. Freeman GJ, Long AJ, Iwai Y, et al. Engagement of the PD-1 immunoinhibitory receptor by a novel B7 family member leads to negative regulation of lymphocyte activation. J Exp Med 2000;192:1027-34.

6. Latchman Y, Wood CR, Chernova T, et al. PD-L2 is a second ligand for PD-1 and inhibits T cell activation. Nat Immunol 2001;2:261-8.

7. Ishida M, Iwai Y, Tanaka Y, et al. Differential expression of PD-L1 and PD-L2, ligands for an inhibitory receptor PD1 , in the cells of lymphohematopoietic tissues. Immunol Lett 2002;84:57-62.

8. Yamazaki T, Akiba H, Iwai H, et al. Expression of programmed death 1 ligands by murine T cells and APC. J Immunol 2002;169:5538-45.

9. Iwai Y, Terawaki S, Honjo T. PD-1 blockade inhibits hematogenous spread of poorly immunogenic tumor cells by enhanced recruitment of effector T cells. Int Immunol 2005;17:133-44.

10. Jain P, Jain C, Velcheti V. Role of immune-checkpoint 
inhibitors in lung cancer. Ther Adv Respir Dis 2018;12:1753465817750075.

11. Horn L, Spigel DR, Vokes EE, et al. Nivolumab Versus Docetaxel in Previously Treated Patients With Advanced Non-Small-Cell Lung Cancer: Two-Year Outcomes From Two Randomized, Open-Label, Phase III Trials (CheckMate 017 and CheckMate 057). J Clin Oncol 2017;35:3924-33.

12. Fehrenbacher L, Spira A, Ballinger M, et al. Atezolizumab versus docetaxel for patients with previously treated non-small-cell lung cancer (POPLAR): a multicentre, open-label, phase 2 randomised controlled trial. Lancet 2016;387:1837-46.

13. Rittmeyer A, Barlesi F, Waterkamp D, et al. Atezolizumab versus docetaxel in patients with previously treated non-small-cell lung cancer (OAK): a phase 3, openlabel, multicentre randomised controlled trial. Lancet 2017;389:255-65.

14. Carbone DP, Reck M, Paz-Ares L, et al. First-Line Nivolumab in Stage IV or Recurrent Non-Small-Cell Lung Cancer. N Engl J Med 2017;376:2415-26.

15. Reck M, Rodriguez-Abreu D, Robinson AG, et al. Pembrolizumab versus Chemotherapy for PD-L1Positive Non-Small-Cell Lung Cancer. N Engl J Med 2016;375:1823-33.

16. Ribas A, Wolchok JD. Cancer immunotherapy using checkpoint blockade. Science 2018;359:1350-5.

17. Schmidt C. The benefits of immunotherapy combinations. Nature 2017;552:S67-9.

18. Borghaei H, Langer CJ, Gadgeel S, et al. LBA49 Updated results from KEYNOTE-021 cohort G: A randomized, phase 2 study of pemetrexed and carboplatin (PC) with or without pembrolizumab (pembro) as first-line therapy for advanced nonsquamous NSCLC. Ann Oncol 2017;28:mdx440.052.

19. Gandhi L, Rodriguez-Abreu D, Gadgeel S, et al. Pembrolizumab plus Chemotherapy in Metastatic Non-Small-Cell Lung Cancer. N Engl J Med 2018;378:2078-92.

20. Vanella V, Festino L, Strudel M, et al. PD-L1 inhibitors in the pipeline: Promise and progress. Oncoimmunology 2017;7:e1365209.

21. Doroshow DB, Herbst RS. Treatment of Advanced Non-Small Cell Lung Cancer in 2018. JAMA Oncol 2018;4:569-70.

22. Champiat S, Dercle L, Ammari S, et al. Hyperprogressive Disease Is a New Pattern of Progression in Cancer Patients Treated by Anti-PD-1/PD-L1. Clin Cancer Res
2017;23:1920-8.

23. Lee CK, Man J, Lord S, et al. Clinical and Molecular Characteristics Associated With Survival Among Patients Treated With Checkpoint Inhibitors for Advanced NonSmall Cell Lung Carcinoma: A Systematic Review and Meta-analysis. JAMA Oncol 2018;4:210-6.

24. Hirsch FR, McElhinny A, Stanforth D, et al. PDL1 Immunohistochemistry Assays for Lung Cancer: Results from Phase 1 of the Blueprint PD-L1 IHC Assay Comparison Project. J Thorac Oncol 2017;12:208-22.

25. Yarchoan M, Hopkins A, Jaffee EM. Tumor Mutational Burden and Response Rate to PD-1 Inhibition. N Engl J Med 2017;377:2500-1.

26. Hellmann MD, Callahan MK, Awad MM, et al. Tumor Mutational Burden and Efficacy of Nivolumab Monotherapy and in Combination with Ipilimumab in Small-Cell Lung Cancer. Cancer Cell 2018;33:853-61.e4.

27. Rizvi NA, Hellmann MD, Snyder A, et al. Cancer immunology. Mutational landscape determines sensitivity to PD-1 blockade in non-small cell lung cancer. Science 2015;348:124-8.

28. Rizvi H, Sanchez-Vega F, La K, et al. Molecular Determinants of Response to Anti-Programmed Cell Death (PD)-1 and Anti-Programmed Death-Ligand 1 (PD-L1) Blockade in Patients With Non-Small-Cell Lung Cancer Profiled With Targeted Next-Generation Sequencing. J Clin Oncol 2018;36:633-41.

29. Karachaliou N, Sosa AE, Molina MA, et al. Possible application of circulating free tumor DNA in non-small cell lung cancer patients. J Thorac Dis 2017;9:S1364-72.

30. Hellmann MD, Nathanson T, Rizvi H, et al. Genomic Features of Response to Combination Immunotherapy in Patients with Advanced Non-Small-Cell Lung Cancer. Cancer Cell 2018;33:843-52.e4.

31. Hellmann MD, Ciuleanu TE, Pluzanski A, et al. Nivolumab plus Ipilimumab in Lung Cancer with a High Tumor Mutational Burden. N Engl J Med 2018;378:2093-104.

32. Khagi Y, Goodman AM, Daniels GA, et al. Hypermutated Circulating Tumor DNA: Correlation with Response to Checkpoint Inhibitor-Based Immunotherapy. Clin Cancer Res 2017;23:5729-36.

33. Kuziora MA, Higgs BW, Brohawn P, et al. Abstract 582: Circulating tumor DNA (ctDNA) variant allele frequencies are reduced in responders to durvalumab and low baseline variant allele frequencies are associated with improved overall survival in NSCLC patients. Cancer Research 2017;77:582. 
34. Kuziora M, Higgs BW, Brohawn PZ, et al. Association of early reduction in circulating tumor DNA (ctDNA) with improved progression-free survival (PFS) and overall survival (OS) of patients (pts) with urothelial bladder cancer (UBC) treated with durvalumab (D). J Clin Oncol 2017;35:11538.

35. Gandara DR, Kowanetz M, Mok TSK, et al. 1295 O Bloodbased biomarkers for cancer immunotherapy: Tumor mutational burden in blood (bTMB) is associated with improved atezolizumab (atezo) efficacy in 2L+ NSCLC (POPLAR and OAK). Ann Oncol 2017;28:mdx380-mdx.

36. Goodman AM, Kato S, Bazhenova L, et al. Tumor Mutational Burden as an Independent Predictor of Response to Immunotherapy in Diverse Cancers. Mol Cancer Ther 2017;16:2598-08.

37. Le DT, Durham JN, Smith KN, et al. Mismatch repair deficiency predicts response of solid tumors to PD-1 blockade. Science 2017;357:409-13.

38. Le DT, Uram JN, Wang H, et al. PD-1 Blockade in Tumors with Mismatch-Repair Deficiency. N Engl J Med 2015;372:2509-20.

39. Schreiber RD, Old LJ, Smyth MJ. Cancer immunoediting: integrating immunity's roles in cancer suppression and promotion. Science 2011;331:1565-70.

40. Karachaliou N, Gonzalez-Cao M, Crespo G, et al. Interferon gamma, an important marker of response to immune checkpoint blockade in non-small cell lung cancer and melanoma patients. Ther Adv Med Oncol 2018;10:1758834017749748.

41. Burr ML, Sparbier CE, Chan YC, et al. CMTM6 maintains the expression of PD-L1 and regulates antitumour immunity. Nature 2017;549:101-5.

42. Mezzadra R, Sun C, Jae LT, et al. Identification of CMTM6 and CMTM4 as PD-L1 protein regulators. Nature 2017;549:106-10.

43. Ceeraz S, Nowak EC, Noelle RJ. B7 family checkpoint regulators in immune regulation and disease. Trends Immunol 2013;34:556-63.

44. Dempke WCM, Fenchel K, Uciechowski P, et al. Second- and third-generation drugs for immunooncology treatment-The more the better? Eur J Cancer 2017;74:55-72.

45. Karachaliou N, Chaib I, Cardona AF, et al. Common Co-activation of AXL and CDCP1 in EGFR-mutationpositive Non-smallcell Lung Cancer Associated With Poor Prognosis. EBioMedicine 2018;29:112-27.

46. Goel S, DeCristo MJ, Watt AC, et al. CDK4/6 inhibition triggers anti-tumour immunity. Nature 2017;548:471-5.
47. Schaer DA, Beckmann RP, Dempsey JA, et al. The CDK4/6 Inhibitor Abemaciclib Induces a T Cell Inflamed Tumor Microenvironment and Enhances the Efficacy of PD-L1 Checkpoint Blockade. Cell Rep 2018;22:2978-94.

48. Lu X, Horner JW, Paul E, et al. Effective combinatorial immunotherapy for castration-resistant prostate cancer. Nature 2017;543:728-32.

49. Ridker PM, MacFadyen JG, Thuren T, et al. Effect of interleukin-1beta inhibition with canakinumab on incident lung cancer in patients with atherosclerosis: exploratory results from a randomised, double-blind, placebocontrolled trial. Lancet 2017;390:1833-42.

50. Huang $\mathrm{Y}$, Jiang $\mathrm{H}$, Chen $\mathrm{Y}$, et al. Tranilast directly targets NLRP3 to treat inflammasome-driven diseases. EMBO Mol Med. 2018;10. doi: 10.15252/emmm.201708689.

51. Ravi R, Noonan KA, Pham V, et al. Bifunctional immune checkpoint-targeted antibody-ligand traps that simultaneously disable TGFbeta enhance the efficacy of cancer immunotherapy. Nat Commun 2018;9:741.

52. Tauriello DVF, Palomo-Ponce S, Stork D, et al. TGF $\beta$ drives immune evasion in genetically reconstituted colon cancer metastasis. Nature 2018;554:538-43.

53. Mariathasan S, Turley SJ, Nickles D, et al. TGFbeta attenuates tumour response to $\mathrm{PD}-\mathrm{L} 1$ blockade by contributing to exclusion of T cells. Nature 2018;554:544-8.

54. Lan Y, Zhang D, Xu C, et al. Enhanced preclinical antitumor activity of $\mathrm{M7824}$, a bifunctional fusion protein simultaneously targeting PD-L1 and TGF-beta. Sci Transl Med. 2018;10. doi: 10.1126/scitranslmed.aan5488.

55. Grinberg-Bleyer Y, Oh H, Desrichard A, et al. NF-kappaB c-Rel Is Crucial for the Regulatory T Cell Immune Checkpoint in Cancer. Cell 2017;170:1096-108.e13.

56. Chamoto K, Chowdhury PS, Kumar A, et al. Mitochondrial activation chemicals synergize with surface receptor PD-1 blockade for T cell-dependent antitumor activity. Proc Natl Acad Sci U S A 2017;114:E761-70.

57. Mao Y, Li W, Chen K, et al. B7-H1 and B7-H3 are independent predictors of poor prognosis in patients with non-small cell lung cancer. Oncotarget 2015;6:3452-61.

58. Inamura K, Yokouchi $Y$, Kobayashi $M$, et al. Tumor B7-H3 (CD276) expression and smoking history in relation to lung adenocarcinoma prognosis. Lung Cancer 2017;103:44-51.

59. Lee YH, Martin-Orozco N, Zheng P, et al. Inhibition of the B7-H3 immune checkpoint limits tumor growth by enhancing cytotoxic lymphocyte function. Cell Res 2017;27:1034-45. 
60. Castellanos JR, Purvis IJ, Labak CM, et al. B7-H3 role in the immune landscape of cancer. Am J Clin Exp Immunol 2017;6:66-75.

61. Zhang M, Li G, Wang Y, et al. PD-L1 expression in lung cancer and its correlation with driver mutations: a metaanalysis. Sci Rep 2017;7:10255.

62. Chen N, Fang W, Zhan J, et al. Upregulation of PDL1 by EGFR Activation Mediates the Immune Escape in EGFR-Driven NSCLC: Implication for Optional Immune Targeted Therapy for NSCLC Patients with EGFR Mutation. J Thorac Oncol 2015;10:910-23.

63. D'Incecco A, Andreozzi M, Ludovini V, et al. PD-1 and PD-L1 expression in molecularly selected non-small-cell lung cancer patients. Br J Cancer 2015;112:95-102.

64. Akbay EA, Koyama S, Carretero J, et al. Activation of the PD-1 pathway contributes to immune escape in EGFRdriven lung tumors. Cancer Discov 2013;3:1355-63.

65. Hong S, Chen N, Fang W, et al. Upregulation of PDL1 by EML4-ALK fusion protein mediates the immune escape in ALK positive NSCLC: Implication for optional anti-PD-1/PD-L1 immune therapy for ALK-TKIs sensitive and resistant NSCLC patients. Oncoimmunology 2015;5:e1094598.

66. Ota K, Azuma K, Kawahara A, et al. Induction of PDL1 Expression by the EML4-ALK Oncoprotein and Downstream Signaling Pathways in Non-Small Cell Lung Cancer. Clin Cancer Res 2015;21:4014-21.

67. Soo RA, Lim SM, Syn NL, et al. Immune checkpoint inhibitors in epidermal growth factor receptor mutant non-small cell lung cancer: Current controversies and future directions. Lung Cancer 2018;115:12-20.

68. Roussel H, De Guillebon E, Biard L, et al. Composite biomarkers defined by multiparametric immunofluorescence analysis identify ALK-positive adenocarcinoma as a potential target for immunotherapy. Oncoimmunology 2017;6:e1286437.

69. Karachaliou N, Cardona A, Cao MG, et al. P2.02-034 PD-L1 Expression Can Be a Prognostic Marker in EGFR Mutant NSCLC Patients Treated with Erlotinib. Journal of Thoracic Oncology;12:S2110-1.

70. Chaib I, Karachaliou N, Pilotto S, et al. Co-activation of STAT3 and YES-Associated Protein 1 (YAP1) Pathway in EGFR-Mutant NSCLC. J Natl Cancer Inst 2017;109. doi: 10.1093/jnci/djx014.

71. Karachaliou N, Giménez-Capitán A, Drozdowskyj A, et al. 8P Expression of genes associated with anti-viral response in EGFR mutant non-small cell lung cancer (NSCLC). Ann Oncol 2017;28:mdx090.008.
72. Wang G, Lu X, Dey P, et al. Targeting YAP-Dependent MDSC Infiltration Impairs Tumor Progression. Cancer Discov 2016;6:80-95.

73. Lee CK, Man J, Lord S, et al. Checkpoint Inhibitors in Metastatic EGFR-Mutated Non-Small Cell Lung Cancer-A Meta-Analysis. J Thorac Oncol 2017;12:403-7.

74. Socinski MA, Jotte RM, Cappuzzo F, et al. Atezolizumab for First-Line Treatment of Metastatic Nonsquamous NSCLC. N Engl J Med 2018;378:2288-301.

75. Sharma P, Hu-Lieskovan S, Wargo JA, et al. Primary, Adaptive, and Acquired Resistance to Cancer Immunotherapy. Cell 2017;168:707-23.

76. Highfill SL, Cui Y, Giles AJ, et al. Disruption of CXCR2mediated MDSC tumor trafficking enhances anti-PD1 efficacy. Sci Transl Med 2014;6:237ra67.

77. Taube JM, Young GD, McMiller TL, et al. Differential Expression of Immune-Regulatory Genes Associated with PD-L1 Display in Melanoma: Implications for PD-1 Pathway Blockade. Clin Cancer Res 2015;21:3969-76.

78. Uehara J, Ohkuri T, Kosaka A, et al. Intratumoral injection of IFN-beta induces chemokine production in melanoma and augments the therapeutic efficacy of anti-PD-L1 mAb. Biochem Biophys Res Commun 2017;490:521-7.

79. Peng D, Kryczek I, Nagarsheth N, et al. Epigenetic silencing of TH1-type chemokines shapes tumour immunity and immunotherapy. Nature 2015;527:249-53.

80. Tripathi SC, Peters HL, Taguchi A, et al. Immunoproteasome deficiency is a feature of non-small cell lung cancer with a mesenchymal phenotype and is associated with a poor outcome. Proc Natl Acad Sci U S A 2016;113:E1555-64.

81. Yarchoan M, Johnson BA 3rd, Lutz ER, et al. Targeting neoantigens to augment antitumour immunity. Nat Rev Cancer 2017;17:569.

82. Hugo W, Zaretsky JM, Sun L, et al. Genomic and Transcriptomic Features of Response to Anti-PD-1 Therapy in Metastatic Melanoma. Cell 2016;165:35-44.

83. Tu S, Bhagat G, Cui G, et al. Overexpression of interleukin-1beta induces gastric inflammation and cancer and mobilizes myeloid-derived suppressor cells in mice. Cancer Cell 2008;14:408-19.

84. Ridker PM, Everett BM, Thuren T, et al. Antiinflammatory Therapy with Canakinumab for Atherosclerotic Disease. N Engl J Med 2017;377:1119-31.

85. Pitt JM, Vetizou M, Daillere R, et al. Resistance Mechanisms to Immune-Checkpoint Blockade in Cancer: Tumor-Intrinsic and -Extrinsic Factors. Immunity 2016;44:1255-69. 
86. Chow MT, Sceneay J, Paget C, et al. NLRP3 suppresses NK cell-mediated responses to carcinogen-induced tumors and metastases. Cancer Res 2012;72:5721-32.

87. van Deventer HW, Burgents JE, Wu QP, et al. The inflammasome component NLRP3 impairs antitumor vaccine by enhancing the accumulation of tumorassociated myeloid-derived suppressor cells. Cancer Res 2010;70:10161-9.

88. Christ A, Gunther P, Lauterbach MAR, et al. Western Diet Triggers NLRP3-Dependent Innate Immune Reprogramming. Cell 2018;172:162-75.e14.

89. Antonia SJ, Villegas A, Daniel D, et al. Durvalumab after Chemoradiotherapy in Stage III Non-Small-Cell Lung Cancer. N Engl J Med 2017;377:1919-29.

90. Venkatesan P. Durvalumab lengthens survival in patients with NSCLC. Lancet Respir Med 2017;5:850.

91. Forde PM, Chaft JE, Smith KN, et al. Neoadjuvant PD-1 Blockade in Resectable Lung Cancer. N Engl J Med 2018;378:1976-86.

92. Melero I, Berraondo P, Rodriguez-Ruiz ME, et al. Making the Most of Cancer Surgery with Neoadjuvant Immunotherapy. Cancer Discov 2016;6:1312-4.

93. Liu J, Blake SJ, Yong MC, et al. Improved Efficacy of Neoadjuvant Compared to Adjuvant Immunotherapy to Eradicate Metastatic Disease. Cancer Discov

Cite this article as: Karachaliou N, Fernandez-Bruno M, Bracht JW, Rosell R. Challenges and unanswered questions for the next decade of immune-oncology research in NSCLC. Transl Lung Cancer Res 2018;7(6):691-702. doi: 10.21037/ tlcr.2018.06.08
2016;6:1382-99.

94. Gettinger S, Horn L, Jackman D, et al. Five-Year FollowUp of Nivolumab in Previously Treated Advanced NonSmall-Cell Lung Cancer: Results From the CA209-003 Study. J Clin Oncol 2018;36:1675-84.

95. Postow MA, Sidlow R, Hellmann MD. Immune-Related Adverse Events Associated with Immune Checkpoint Blockade. N Engl J Med 2018;378:158-68.

96. Sosa AE, Lopez CE, Simon OC, et al. Clinical assessment of immune-related adverse events. Ther Adv Med Oncol 2018;10:1758835918764628.

97. Fruh M, Peters S. Genomic Features of Response to Combination Immunotherapy in Lung Cancer. Cancer Cell 2018;33:791-3.

98. Alexander BM, Schoenfeld JD, Trippa L. Hazards of Hazard Ratios - Deviations from Model Assumptions in Immunotherapy. N Engl J Med 2018;378:1158-9.

99. Langer CJ, Gadgeel SM, Borghaei H, et al. Carboplatin and pemetrexed with or without pembrolizumab for advanced, non-squamous non-small-cell lung cancer: a randomised, phase 2 cohort of the open-label KEYNOTE-021 study. Lancet Oncol 2016;17:1497-508.

100.Huang AC, Postow MA, Orlowski RJ, et al. T-cell invigoration to tumour burden ratio associated with antiPD-1 response. Nature 2017;545:60-5. 